Article

\title{
Relativistic Combination of Non-Collinear 3-Velocities Using Quaternions
}

\author{
Thomas Berry ${ }^{*}+{ }^{(D)}$ and Matt Visser ${ }^{+}$(D) \\ School of Mathematics and Statistics, Victoria University of Wellington, PO Box 600, \\ Wellington 6140, New Zealand; matt.visser@sms.vuw.ac.nz \\ * Correspondence: thomas.berry@sms.vuw.ac.nz \\ t These authors contributed equally to this work.
}

Received: 18 November 2020; Accepted: 8 December 2020; Published: 11 December 2020

\begin{abstract}
Quaternions have an (over a century-old) extensive and quite complicated interaction with special relativity. Since quaternions are intrinsically 4-dimensional, and do such a good job of handling 3-dimensional rotations, the hope has always been that the use of quaternions would simplify some of the algebra of the Lorentz transformations. Herein we report a new and relatively nice result for the relativistic combination of non-collinear 3-velocities. We work with the relativistic half-velocities $w$ defined by $v=\frac{2 w}{1+w^{2}}$, so that $w=\frac{v}{1+\sqrt{1-v^{2}}}=\frac{v}{2}+\mathcal{O}\left(v^{3}\right)$, and promote them to quaternions using $\mathbf{w}=w \hat{\mathbf{n}}$, where $\hat{\mathbf{n}}$ is a unit quaternion. We shall first show that the composition of relativistic half-velocities is given by $\mathbf{w}_{1 \oplus 2} \equiv \mathbf{w}_{1} \oplus \mathbf{w}_{2} \equiv\left(1-\mathbf{w}_{1} \mathbf{w}_{2}\right)^{-1}\left(\mathbf{w}_{1}+\mathbf{w}_{2}\right)$, and then show that this is also equivalent to $\mathbf{w}_{1 \oplus 2}=\left(\mathbf{w}_{1}+\mathbf{w}_{2}\right)\left(1-\mathbf{w}_{2} \mathbf{w}_{1}\right)^{-1}$. Here as usual we adopt units where the speed of light is set to unity. Note that all of the complicated angular dependence for relativistic combination of non-collinear 3-velocities is now encoded in the quaternion multiplication of $\mathbf{w}_{1}$ with $\mathbf{w}_{2}$. This result can furthermore be extended to obtain novel elegant and compact formulae for both the associated Wigner angle $\Omega$ and the direction of the combined velocities: $\mathrm{e}^{\Omega}=\mathrm{e}^{\Omega \hat{\Omega}}=\left(1-\mathbf{w}_{1} \mathbf{w}_{2}\right)^{-1}\left(1-\mathbf{w}_{2} \mathbf{w}_{1}\right)$, and $\hat{\mathbf{w}}_{1 \oplus 2}=\mathrm{e}^{\Omega / 2} \frac{\mathbf{w}_{1}+\mathbf{w}_{2}}{\left|\mathbf{w}_{1}+\mathbf{w}_{2}\right|}$. Finally, we use this formalism to investigate the conditions under which the relativistic composition of 3-velocities is associative. Thus, we would argue, many key results that are ultimately due to the non-commutativity of non-collinear boosts can be easily rephrased in terms of the non-commutative algebra of quaternions.
\end{abstract}

Keywords: special relativity; combination of velocities; wigner angle; quaternions

\section{Introduction}

Hamilton first described the quaternions in the mid-1800s, primarily with a view to finding algebraically simple ways to handle 3-dimensional rotations. With the advent of special relativity in 1905, and noting the manifestly 4-dimensional nature of quaternions once one adds a real part, multiple authors have tried to interpret special relativity in an intrinsically quaternionic fashion [1-9].

Despite technical success in applying quaternions to special relativity, the use of quaternions in this subject has never really gained all that much traction in the physics community. Perhaps one of the reasons for this is that there are a number of sub-optimal notational choices in Silberstein's original work [1-3], and the fact that there is no generally accepted way of using quaternions to represent Lorentz transformations, with many different authors employing their own quite distinct methods [1-9]. Even in more recent, post-millennial, articles on "quaternionic special relativity" there is considerable disagreement on notational choices [10-13].

Below we shall introduce what we feel is a particularly simple and straightforward method for combining relativistic 3-velocities using quaternions. In particular, we shall present some new and compact formulae for computing the Wigner angle [14]. All of the interesting features due to 
non-commutativity properties of non-collinear boosts are implicitly and rather efficiently dealt with by the non-commutative algebra of quaternions. The method is based on an extension of an analysis by Giust, Vigoureux, and Lages $[15,16]$, who (because they were working with the usual complex numbers) were essentially limited to motion in 2-space; their formalism is not really well-adapted to general motions in 3-space. Related constructions can also be found in [10,11].

Observe that there is a representation of pure quaternions in terms of a subset of $2 \times 2$ matrices, specifically the anti-hermitian $2 \times 2$ matrices, essentially $\sqrt{-1} \times$ (Pauli matrices). (The factor of $\sqrt{-1}$ is important.) However this does not mean that replacing quaternions by Pauli matrices in any way simplifies our results below; it just complicates the formalism. Neither does this mean that any of our results below are at all "well-known" in this alternate notation. We have carefully checked the relevant literature, (roughly speaking, 2-spinor representations of the Lorentz group). There is much more than pedagogy going on-the results reported in our article are (apart from a consistency check or two) both novel and interesting (see also [13].)

\section{Preliminaries}

\subsection{Lorentz Transformations}

The set of all Lorentz transformations of space-time form a group called the Lorentz group. Mathematically, the Lorentz group is isomorphic to $\mathrm{O}(1,3)$, the orthogonal group of one time and three space dimensions that preserves the space-time interval

$$
s^{2}=-t^{2}+x^{2}+y^{2}+z^{2} .
$$

Here and hereafter, as usual we adopt units where the speed of light is set to unity. It is clear from this description that rotations of space-time are included in the Lorentz group, as well as the more familiar pure Lorentz transformations (boosts). In fact, the pure Lorentz transformations do not even form a subgroup of the Lorentz group as, in general, the composition of two boosts $B_{1}$ and $B_{2}$ is not another boost but in fact a boost and a rotation $B_{12} R_{12}=B_{1} B_{2}$; while $B_{21} R_{21}=B_{2} B_{1}$. This rotation, known as the Wigner rotation, was first discovered by Llewellyn Thomas in 1926 whilst trying to describe the Zeeman effect from a relativistic view-point [17], and was more fully analyzed by Eugene Wigner in 1939 [14]. (For more recent discussions see [18-22]).

It is well-known that the composition of Lorentz transformations is non-commutative. That is, applying two successive boosts $B_{1}$ and $B_{2}$ in different orders results in the same final boost, $B_{12}=B_{21}$, but different rotations, $R_{12} \neq R_{21}$. In the context of the combination of two velocities $\vec{v}_{1}$ and $\vec{v}_{2}$, this means that the final speed is the same no matter the order we combine the velocities, $\left\|\vec{v}_{1} \oplus \vec{v}_{2}\right\|=$ $\left\|\vec{v}_{2} \oplus \vec{v}_{1}\right\|$, but the final directions they point in are different $\hat{v}_{1 \oplus 2} \neq \hat{v}_{2 \oplus 1}$. Although not immediately obvious, the angle between $\vec{v}_{1 \oplus 2}=\vec{v}_{1} \oplus \vec{v}_{2}$ and $\vec{v}_{2 \oplus 1}=\vec{v}_{2} \oplus \vec{v}_{1}$ is in fact the Wigner angle $\Omega$, see Reference [22]. The Lorentz group has very many different representations, one of which is formulated by using the quaternions $[1,2,4]$.

One could instead try to deal with the non-commutativity of the Lorentz transformations by adapting the general formalism of the Baker-Campbell-Hausdorff theorem [23-27]. Unfortunately the general BCH formalism applied to this problem very quickly becomes intractable, and we have found that the specifics of the quaternion formalism yield much more useful and tractable results.

Since the full symmetry group of the Maxwell equations is the conformal extension of the Poincare group, it is sometimes useful, (when looking at pure electromagnetic effects), to work with this conformal extension. However physical observers, (physical clocks and physical rulers), break the conformal invariance, and to even meaningfully define 3-velocities one needs to restrict attention to the Poincare group. We shall go even further and take translation invariance (spatial and temporal homogeneity) for granted, and focus more specifically on the Lorentz group. 


\subsection{Quaternions}

The quaternions are numbers that can be written in the form $a+b \mathbf{i}+c \mathbf{j}+d \mathbf{k}$, where $a, b, c$, and $d$ are real numbers, and $\mathbf{i}, \mathbf{j}$, and $\mathbf{k}$ are the quaternion units which satisfy the famous relation

$$
\mathbf{i}^{2}=\mathbf{j}^{2}=\mathbf{k}^{2}=\mathbf{i j k}=-1 .
$$

They form a four-dimensional number system that is generally treated as an extension of the complex numbers. We shall define the quaternion conjugate of the quaternion $\mathbf{q}=a+b \mathbf{i}+c \mathbf{j}+d \mathbf{k}$ to be $\mathbf{q}^{\star}=a-b \mathbf{i}-c \mathbf{j}-d \mathbf{k}$, and define the norm of $\mathbf{q}$ to be $\mathbf{q} \mathbf{q}^{\star}=|\mathbf{q}|^{2}=a^{2}+b^{2}+c^{2}+d^{2} \in \mathbb{R}^{+}$. This allows us to evaluate the quaternion inverse as $\mathbf{q}^{-1}=\mathbf{q}^{\star} /|\mathbf{q}|^{2}$.

Trying to define a "norm" as $\mathbf{q}^{2}=a^{2}-b^{2}-c^{2}-d^{2}$, while superficially more "relativistic", violates the usual mathematical definition of "norm", and furthermore is not useful when it comes to evaluating the quaternion inverse $\mathbf{q}^{-1}$.

For current purposes we focus our attention on pure quaternions. That is, we consider quaternions of the form $a \mathbf{i}+b \mathbf{j}+c \mathbf{k}$. Many quaternion operations become much simpler when we are dealing with pure quaternions. For example, the product of two pure quaternions $\mathbf{p}$ and $\mathbf{q}$ is given by $\mathbf{p q}=-\vec{p} \cdot \vec{q}+(\vec{p} \times \vec{q}) \cdot(\mathbf{i}, \mathbf{j}, \mathbf{k})$, where, in general, we shall set $\mathbf{v}=\vec{v} \cdot(\mathbf{i}, \mathbf{j}, \mathbf{k})$. From this, we obtain the useful relations

$$
[\mathbf{p}, \mathbf{q}]=2(\vec{p} \times \vec{q}) \cdot(\mathbf{i}, \mathbf{j}, \mathbf{k}) \text {, and }\{\mathbf{p}, \mathbf{q}\}=-2 \vec{p} \cdot \vec{q} .
$$

A notable consequence of (3) is $\mathbf{q}^{2}=-\vec{q} \cdot \vec{q}=-q^{2}=-|\mathbf{q}|^{2}$. There is a natural isomorphism between the space of pure quaternions and $\mathbb{R}^{3}$ given by

$$
\mathbf{i} \mapsto \hat{x}, \quad \mathbf{j} \mapsto \hat{y}, \quad \mathbf{k} \mapsto \hat{z} ;
$$

where $\hat{x}, \hat{y}$, and $\hat{z}$ are the standard unit vectors in $\mathbb{R}^{3}$.

One of the most common uses for quaternions today (2020) is in the computer graphics community, where they are used to compactly and efficiently generate rotations in 3 -space. Indeed, if $\mathbf{q}=\cos (\theta / 2)+\hat{\mathbf{u}} \sin (\theta / 2)$ is an arbitrary unit quaternion and $\mathbf{v}$ is the image of a vector in $\mathbb{R}^{3}$ under the isomorphism (4), then the mapping $\mathbf{v} \mapsto \mathbf{q v q}^{-1}$ rotates $\mathbf{v}$ through an angle $\theta$ about the axis defined by $\hat{\mathbf{u}}$. The mapping $\mathbf{v} \mapsto \mathbf{q v q}^{-1}$ is called quaternion conjugation by $\mathbf{q}$.

\section{Combining Two 3-Velocities}

In the paper by Giust, Vigoureux, and Lages [15], see also Reference [16], (and the somewhat related discussion in Reference [10]), a method is developed to compactly combine relativistic velocities in two space dimensions, and by extension, coplanar relativistic velocities in 3 space dimensions. In the following subsection, we first provide a short summary of their approach, and then in the next subsection extend their method to general non-coplanar 3-velocities.

\subsection{Velocities in the $(x, y)$-Plane}

The success of this Giust, Vigoureux, and Lages approach relies on the angle addition formula for the hyperbolic tangent function,

$$
\tanh \left(\xi_{1}+\xi_{2}\right)=\frac{\tanh \xi_{1}+\tanh \xi_{2}}{1+\tanh \xi_{1} \tanh \xi_{2}} .
$$

The tanh function is a natural choice for combining relativistic velocities since it is limited to the interval $[-1,1]$. Indeed, using the rapidity $\xi$ defined by $v=\tanh (\xi)$, we can easily combine collinear relativistic speeds using Equation (5). In order to use this for the combination of non-collinear relativistic 2-velocities, we replace each 2 -velocity $\vec{v}$ by the complex number

$$
V=\tanh (\xi / 2) \mathrm{e}^{i \varphi}
$$


Here $\xi$ is the rapidity of the velocity $\vec{v}$, and $\varphi$ gives the orientation of $\vec{v}$ according to some observer in the plane defined by $\vec{v}_{1}$ and $\vec{v}_{2}$. Giust, Vigoureux, and Lages then define the composition law $\oplus$ for coplanar velocities $\vec{v}_{1}$ and $\vec{v}_{2}$ by

$$
W=\tanh \frac{\xi}{2} \mathrm{e}^{i \varphi_{1 \oplus 2}}=V_{1} \oplus V_{2}=\frac{V_{1}+V_{2}}{1+\overline{V_{2}} V_{1}}=\frac{\tanh \frac{\xi_{1}}{2} \mathrm{e}^{i \varphi_{1}}+\tanh \frac{\xi_{2}}{2} \mathrm{e}^{i \varphi_{2}}}{1+\tanh \frac{\xi_{2}}{2} \mathrm{e}^{-i \varphi_{2}} \tanh \frac{\xi_{1}}{2} \mathrm{e}^{i \varphi_{1}}},
$$

where $\bar{V}$ is the standard complex conjugate of $V$. By using $\xi / 2$ instead of $\xi$ in Equations (6) and (7), we are actually dealing with the "relativistic half-velocities", $\tanh (\xi / 2)$, (sometimes called the "symmetric velocities"), where

$$
w=\tanh (\xi / 2) ; \quad v=\tanh (\xi)=\frac{2 w}{1+w^{2}} .
$$

That is:

$$
w=\tanh \left(\frac{1}{2} \tanh ^{-1}(v)\right)=\frac{v}{1+\sqrt{1-v^{2}}} .
$$

Using Equations (5) and (7) we can easily retrieve the real velocity from the half-velocity by using the $\oplus$ operator: $v=\tanh \xi=\tanh \xi / 2 \oplus \tanh \xi / 2=w \oplus w$. In terms of the half velocities

$$
w_{1 \oplus 2} \mathrm{e}^{i \varphi_{1 \oplus 2}}=\frac{w_{1} \mathrm{e}^{i \varphi_{1}}+w_{2} \mathrm{e}^{i \varphi_{2}}}{1+w_{1} w_{2} \mathrm{e}^{i\left(\varphi_{1}-\varphi_{2}\right)}} .
$$

The $\oplus$ addition law is non-commutative, which is most easily seen by first setting $\theta=\varphi_{2}-\varphi_{1}$, then $\Omega=\varphi_{1 \oplus 2}-\varphi_{2 \oplus 1}$, and finally observing that the ratio

$$
\mathrm{e}^{i \Omega / 2}=\frac{1+\tanh \frac{\xi_{1}}{2} \tanh \frac{\tilde{\xi}_{2}}{2} \mathrm{e}^{i \theta}}{1+\tanh \frac{\tilde{\xi}_{1}}{2} \tanh \frac{\tilde{\xi}_{2}}{2} \mathrm{e}^{-i \theta}}=\frac{1+w_{1} w_{2} \mathrm{e}^{i \theta}}{1+w_{1} w_{2} \mathrm{e}^{-i \theta}}
$$

is not equal to unity for non-zero $\theta$, meaning that $\Omega=\varphi_{1 \oplus 2}-\varphi_{2 \oplus 1}$ is non-zero.

The angle $\Omega=\varphi_{1 \oplus 2}-\varphi_{2 \oplus 1}$ is in fact the Wigner angle $\Omega$, so an expression for this angle can be obtained by taking the real and imaginary parts of Equation (11):

$$
\tan \frac{\Omega}{2}=\frac{\tanh \frac{\xi_{1}}{2} \tanh \frac{\tilde{\xi}_{2}}{2} \sin \theta}{1+\tanh \frac{\xi_{1}}{2} \tanh \frac{\tilde{\xi}_{2}}{2} \cos \theta}=\frac{w_{1} w_{2} \sin \theta}{1+w_{1} w_{2} \cos \theta} .
$$

This expression does not explicitly appear in Reference [15] though something functionally equivalent, in the form $\Omega=2 \arg \left(1+w_{1} w_{2} e^{i \theta}\right)$, appears in Reference [16].

The $\oplus$ law can be applied to any number of coplanar velocities by iteration:

$$
W=\left(\left(\left(V_{1} \oplus V_{2}\right) \oplus \cdots \oplus V_{n-1}\right) \oplus V_{n}\right) .
$$

Thus it would be desirable to cleanly extend this formalism to general three-dimensional velocities. Note that the order of composition is important, as we shall see in more detail below, the $\oplus$ operation is in general not associative.

\subsection{General 3-Velocities}

We now extend the result of Giust, Vigoureux, and Lages to arbitrary 3-velocities in three dimensions. 


\subsubsection{Algorithm}

Suppose we have a velocity $\vec{v}_{i}$ in the $(x, y)$-plane, represented by the pure quaternion $\mathbf{w}_{i}=\tanh \left(\xi_{i} / 2\right) \hat{\mathbf{n}}_{i}=\tanh \left(\xi_{i} / 2\right)\left(\mathbf{i} \cos \theta_{i}+\mathbf{j} \sin \theta_{i}\right)$. Using the rules for quaternion multiplication, we can write this as $\mathbf{w}_{i}=\tanh \left(\xi_{i} / 2\right)\left(\cos \theta_{i}+\mathbf{k} \sin \theta_{i}\right) \mathbf{i}$. The term inside the brackets now looks very similar to what would be a natural extension of the exponential function to the quaternions, $\mathrm{e}^{\mathbf{k} \theta}=\cos \theta+\mathbf{k} \sin \theta$. To formalise this, we define the exponential of a quaternion $\mathbf{q}$ by the power series

$$
\mathrm{e}^{\mathbf{q}}=\sum_{k=0}^{\infty} \frac{\mathbf{q}^{k}}{k !}
$$

To calculate an explicit formula for Equation (14), we first consider the case of a pure quaternion $\mathbf{u}$. We know from Section 2.2 that for a pure quaternion we have $\mathbf{u}^{2}=-|\mathbf{u}|^{2}$, and so we find $\mathbf{u}^{3}=-|\mathbf{u}|^{2} \mathbf{u}, \mathbf{u}^{4}=|\mathbf{u}|^{4}$, and so on. Thus, we can compute

$$
\mathrm{e}^{\mathbf{u}} \equiv \sum_{k=0}^{\infty} \frac{\mathbf{u}^{k}}{k !}=\left(1-\frac{1}{2 !}|\mathbf{u}|^{2}+\frac{1}{4 !}|\mathbf{u}|^{4}-\ldots\right)+\frac{\mathbf{u}}{|\mathbf{u}|}\left(|\mathbf{u}|-\frac{1}{3 !}|\mathbf{u}|^{3}+\frac{1}{5 !}|\mathbf{u}|^{5}-\ldots\right)=\cos |\mathbf{u}|+\hat{\mathbf{u}} \sin |\mathbf{u}| .
$$

Following the same procedure above, we find the exponential of a pure unit quaternion $\hat{\mathbf{u}}$ and real number $\phi$ to be

$$
\mathrm{e}^{\hat{\mathbf{u}} \phi}=\cos \phi+\hat{\mathbf{u}} \sin \phi
$$

This nice result reflects the expression for the exponential of a complex number.

We can now extend this result to any arbitrary quaternion $\mathbf{q}=a+\mathbf{u}$ by noting that the real number $a$ commutes with all the terms in $\mathbf{u}$, thereby allowing us to write $\mathrm{e}^{\mathbf{q}}=\mathrm{e}^{a} \mathrm{e}^{\mathbf{u}}$, where $\mathrm{e}^{\mathbf{u}}$ has the same form as Equation (15). Explicitly,

$$
\mathrm{e}^{\mathbf{q}}=\mathrm{e}^{a}(\cos |\mathbf{u}|+\hat{\mathbf{u}} \sin |\mathbf{u}|)
$$

The exponential of a quaternion possesses many of the same properties as the exponential of a complex number. Two particularly useful ones we use below are

$$
\left(\mathrm{e}^{\hat{\mathbf{u}} \phi}\right)^{\star}=\mathrm{e}^{-\hat{\mathbf{u}} \phi}=\cos \phi-\hat{\mathbf{u}} \sin \phi, \quad \text { and } \quad\left|\mathrm{e}^{\hat{\mathbf{u}} \phi}\right|=1 .
$$

Using these results, we are now justified in writing

$$
\mathbf{w}_{i}=\tanh \left(\xi_{i} / 2\right) \mathrm{e}^{\mathbf{k} \theta_{i}} \mathbf{i}=w_{i} \mathrm{e}^{\mathbf{k} \theta_{i}} \mathbf{i}
$$

for our velocity in the $(x, y)$-plane.

Building on this result, we now find it appropriate to define the $\oplus$ operator for general 3-velocities, $\mathbf{w}_{1}=w_{1} \hat{\mathbf{n}}_{\mathbf{1}}$ and $\mathbf{w}_{2}=w_{2} \hat{\mathbf{n}}_{\mathbf{2}}$, by the novel formula:

$$
\mathbf{w}_{1 \oplus 2}=\mathbf{w}_{1} \oplus \mathbf{w}_{2}=\left(1-\mathbf{w}_{1} \mathbf{w}_{2}\right)^{-1}\left(\mathbf{w}_{1}+\mathbf{w}_{2}\right) .
$$

The usefulness of this novel definition is best understood by looking at a few examples.

\subsubsection{Example: Parallel Velocities}

We consider two parallel velocities $\vec{v}_{1}$ and $\vec{v}_{2}$ represented by the quaternions

$$
\mathbf{w}_{1}=\tanh \frac{\xi_{1}}{2} \hat{\mathbf{n}} \quad \text { and } \quad \mathbf{w}_{2}=\tanh \frac{\xi_{2}}{2} \hat{\mathbf{n}},
$$


respectively. Our composition law (20) then gives

$$
\begin{aligned}
\mathbf{w}_{1 \oplus 2} & =\left(1+\tanh \frac{\xi_{1}}{2} \tanh \frac{\tilde{\xi}_{2}}{2}\right)^{-1}\left(\tanh \frac{\xi_{1}}{2} \hat{\mathbf{n}}+\tanh \frac{\xi_{2}}{2} \hat{\mathbf{n}}\right) \\
& =\frac{\tanh \frac{\xi_{1}}{2}+\tanh \frac{\xi_{2}}{2}}{1+\tanh \hat{\xi_{1}} \tanh \frac{\xi_{2}}{2}} \hat{\mathbf{n}} \\
& =\tanh \left(\frac{\tilde{\xi}_{1}+\xi_{2}}{2}\right) \hat{\mathbf{n}},
\end{aligned}
$$

which is equivalent to

$$
\mathbf{w}_{1 \oplus 2}=\frac{w_{1}+w_{2}}{1+w_{1} w_{2}} \hat{\mathbf{n}},
$$

and hence, also equivalent to the well-known result for the relativistic composition of two parallel velocities,

$$
\vec{v}_{1} \oplus \vec{v}_{2}=\frac{v_{1}+v_{2}}{1+v_{1} v_{2}} \hat{n}
$$

\subsubsection{Example: Perpendicular Velocities in the $x-y$ Plane}

We now consider two perpendicular velocities in the $x-y$ plane. By rotating around the $z$ axis, without loss of generality they can be taken to be given by

$$
\mathbf{w}_{1}=w_{1} \mathbf{i}, \quad \mathbf{w}_{2}=w_{2} \mathbf{j},
$$

where we have written $\tanh \left(\xi_{1} / 2\right)=w_{1}$ and $\tanh \left(\xi_{2} / 2\right)=w_{2}$ for brevity.

Our composition law then gives a combined velocity of

$$
\mathbf{w}_{1 \oplus 2}=\left(1-w_{1} w_{2} \mathbf{i} \mathbf{j}\right)^{-1}\left(w_{1} \mathbf{i}+w_{2} \mathbf{j}\right)=\frac{w_{1}\left(1-w_{2}^{2}\right) \mathbf{i}+w_{2}\left(1+w_{1}^{2}\right) \mathbf{j}}{1+w_{1}^{2} w_{2}^{2}},
$$

which is definitely not commutative. In contrast the norm is symmetric:

$$
\left|\mathbf{w}_{1 \oplus 2}\right|^{2}=\frac{w_{1}^{2}\left(1-w_{2}^{2}\right)^{2}+w_{2}^{2}\left(1+w_{1}^{2}\right)^{2}}{\left(1+w_{1}^{2} w_{2}^{2}\right)^{2}}=\frac{w_{1}^{2}+w_{2}^{2}+w_{1}^{2} w_{2}^{4}+w_{2}^{2} w_{1}^{4}}{\left(1+w_{1}^{2} w_{2}^{2}\right)^{2}}=\frac{w_{1}^{2}+w_{2}^{2}}{1+w_{1}^{2} w_{2}^{2}} .
$$

Here the $\mathbf{w}_{i}$ are the "relativistic half-velocities" $w_{i}=\tanh \left(\xi_{i} / 2\right)$, so the full velocities are

$$
\left|\mathbf{v}_{i}\right|^{2}=\left|\mathbf{w}_{i} \oplus \mathbf{w}_{i}\right|^{2}=\frac{4 w_{i}^{2}}{\left(1+w_{i}^{2}\right)^{2}}
$$

and so give a final speed of

$$
\left|\mathbf{v}_{1 \oplus 2}\right|^{2}=\frac{4\left(w_{1}^{2}+w_{2}^{2}\right)}{\left(1+w_{1}^{2} w_{2}^{2}\right)\left[1+\frac{w_{1}^{2}+w_{2}^{2}}{1+w_{1}^{2} w_{2}^{2}}\right]^{2}}=\frac{4\left(w_{1}^{2}+w_{2}^{2}\right)\left(1+w_{1}^{2} w_{2}^{2}\right)}{\left[\left(1+w_{1}^{2}\right)\left(1+w_{2}^{2}\right)\right]^{2}} .
$$

The non-quaternionic result for the composition of two perpendicular velocities is [22]

$$
\left\|\vec{v}_{1 \oplus 2}\right\|^{2}=v_{1}^{2}+v_{2}^{2}-v_{1}^{2} v_{2}^{2} .
$$

Thus, we find

$$
\left\|\vec{v}_{1 \oplus 2}\right\|^{2}=\frac{4 w_{1}^{2}}{\left(1+w_{1}^{2}\right)^{2}}+\frac{4 w_{2}^{2}}{\left(1+w_{2}^{2}\right)^{2}}-\frac{16 w_{1}^{2} w_{2}^{2}}{\left(1+w_{1}^{2}\right)^{2}\left(1+w_{2}^{2}\right)^{2}}=\frac{4\left(w_{1}^{2}+w_{2}^{2}\right)\left(1+w_{1}^{2} w_{2}^{2}\right)}{\left[\left(1+w_{1}^{2}\right)\left(1+w_{2}^{2}\right)\right]^{2}} .
$$


And so our composition law $\oplus$ gives the standard result for the composition of two perpendicular velocities in the $x-y$ plane.

\subsubsection{Example: Perpendicular Velocities in General}

For general perpendicular velocities $\vec{v}_{1}$ and $\vec{v}_{2}$ the easiest way of proceeding is to simply rotate to point $\vec{v}_{1}$ along the $x$-axis and $\vec{v}_{2}$ along the $y$-axis, and just copy the argument above. If one wishes to be more direct then simply define

$$
\mathbf{w}_{1}=w_{1} \widehat{\mathbf{w}_{1}}, \quad \mathbf{w}_{2}=w_{2} \widehat{\mathbf{w}_{2}} ; \quad \widehat{\mathbf{w}_{3}}=\widehat{\mathbf{w}_{1}} \widehat{\mathbf{w}_{2}} .
$$

In view of the mutual orthogonality of the vectors $\hat{w}_{1}, \hat{w}_{2}$, and $\hat{w}_{3}$, the unit quaternions $\left(\widehat{\mathbf{w}_{1}}, \widehat{\mathbf{w}_{2}}, \widehat{\mathbf{w}_{3}}\right)$ obey exactly the same commutation relations as $(\mathbf{i}, \mathbf{j}, \mathbf{k})$. Thence

$$
\mathbf{w}_{1 \oplus 2}=\left(1-w_{1} w_{2} \widehat{\mathbf{w}_{1}} \widehat{\mathbf{w}}_{2}\right)^{-1}\left(w_{1} \widehat{\mathbf{w}_{1}}+w_{2} \widehat{\mathbf{w}_{2}}\right)=\frac{w_{1}\left(1-w_{2}^{2}\right) \widehat{\mathbf{w}_{1}}+w_{2}\left(1+w_{1}^{2}\right) \widehat{\mathbf{w}_{2}}}{1+w_{1}^{2} w_{2}^{2}} .
$$

This now leads to exactly the same results as above; there was no loss of generality inherent in working in the $x-y$ plane.

\subsubsection{Example: Reduction to Giust-Vigoureux-Lages Result in the $x-y$ Plane}

It is important to note that our composition law $\oplus$ reduces to the composition law of Giust, Vigoureux, and Lages [15] when dealing with planar velocities in the $x-y$ plane. As above, we define general velocities in the (i, $\mathbf{j})$-plane by $\mathbf{w}_{1}=\tanh \left(\xi_{1} / 2\right) \mathrm{e}^{\mathbf{k} \phi_{1}} \mathbf{i}$, and $\mathbf{w}_{2}=\tanh \left(\xi_{2} / 2\right) \mathrm{e}^{\mathbf{k} \phi_{2}} \mathbf{i}$, then, using our composition law (20), we find

$$
\mathbf{w}_{1 \oplus 2}=\left(1-\tanh \frac{\xi_{1}}{2} \mathrm{e}^{\mathbf{k} \phi_{1}} \mathbf{i} \tanh \frac{\xi_{2}}{2} \mathrm{e}^{\mathbf{k} \phi_{2}} \mathbf{i}\right)^{-1}\left(\tanh \frac{\xi_{1}}{2} \mathrm{e}^{\mathbf{k} \phi_{1}} \mathbf{i}+\tanh \frac{\xi_{2}}{2} \mathrm{e}^{\mathbf{k} \phi_{2}} \mathbf{i}\right) .
$$

But, noting that $\tanh \left(\xi_{2} / 2\right) \mathrm{e}^{\mathbf{k} \phi_{2}} \mathbf{i}=\tanh \left(\xi_{2} / 2\right) \mathbf{i} \mathrm{e}^{-\mathbf{k} \phi_{2}}$ and $\mathbf{i}^{2}=-1$, we can re-write this as

$$
\mathbf{w}_{1 \oplus 2}=\left(1+\tanh \frac{\xi_{1}}{2} \mathrm{e}^{\mathbf{k} \phi_{1}} \tanh \frac{\xi_{2}}{2} \mathrm{e}^{-\mathbf{k} \phi_{2}}\right)^{-1}\left(\tanh \frac{\xi_{1}}{2} \mathrm{e}^{\mathbf{k} \phi_{1}}+\tanh \frac{\xi_{2}}{2} \mathrm{e}^{\mathbf{k} \phi_{2}}\right) \mathbf{i}
$$

Now writing

$$
\mathbf{w}_{1 \oplus 2}=\tanh \left(\xi_{1 \oplus 2} / 2\right) \mathrm{e}^{\mathbf{k} \phi_{1 \oplus 2}} \mathbf{i}
$$

we can cancel out the trailing $\mathbf{i}$, to obtain

$$
\tanh \frac{\xi_{1 \oplus 2}}{2} \mathrm{e}^{\mathbf{k} \phi_{1 \oplus 2}}=\left(1+\tanh \frac{\xi_{1}}{2} \mathrm{e}^{\mathbf{k} \phi_{1}} \tanh \frac{\xi_{2}}{2} \mathrm{e}^{-\mathbf{k} \phi_{2}}\right)^{-1}\left(\tanh \frac{\xi_{1}}{2} \mathrm{e}^{\mathbf{k} \phi_{1}}+\tanh \frac{\xi_{2}}{2} \mathrm{e}^{\mathbf{k} \phi_{2}}\right) .
$$

This expression now only contains $\mathbf{k}$, so everything commutes, and we can write

$$
w_{1 \oplus 2} \mathrm{e}^{\mathbf{k} \phi_{1 \oplus 2}}=\frac{w_{1} \mathrm{e}^{\mathbf{k} \phi_{1}}+w_{2} \mathrm{e}^{\mathbf{k} \phi_{2}}}{1+w_{1} \mathrm{e}^{\mathbf{k} \phi_{1}} w_{2} \mathrm{e}^{-\mathbf{k} \phi_{2}}}
$$

which is equivalent to the result of Giust, Vigoureux, and Lages [15]. 


\subsubsection{Example: Composition in General Directions}

For general velocities $\vec{v}_{1}$ and $\vec{v}_{2}$ the easiest way of proceeding is to simply rotate to put $\vec{v}_{1}$ and $\vec{v}_{2}$ in the the $x-y$ plane, and just copy the Giust-Vigoureux-Lages argument [15] above. If one wishes to be more direct then simply define

$$
\mathbf{w}_{1}=w_{1} \widehat{\mathbf{w}_{1}}, \quad \mathbf{w}_{2}=w_{2} \widehat{\mathbf{w}_{2}} ; \quad \widehat{\mathbf{w}_{3}}=\frac{\left[\widehat{\mathbf{w}_{1}}, \widehat{\mathbf{w}_{2}}\right]}{\left|\left[\widehat{\mathbf{w}}_{1}, \widehat{\mathbf{w}_{2}}\right]\right|} .
$$

As long as $\widehat{\mathbf{w}_{1}}$ is not parallel to $\widehat{\mathbf{w}_{2}}$, then $\widehat{\mathbf{w}_{3}}$ is well defined and perpendicular to both $\widehat{\mathbf{w}_{1}}$ and $\widehat{\mathbf{w}_{2}}$. With these definitions one can now write

$$
\widehat{\mathbf{w}_{2}}=\exp \left(\phi \widehat{\mathbf{w}_{3}}\right) \widehat{\mathbf{w}_{1}} .
$$

Then, following the discussion above, we see

$$
\mathbf{w}_{1 \oplus 2}=\left(1+w_{1} w_{2} \mathrm{e}^{-\widehat{\mathbf{w}_{3}} \phi}\right)^{-1}\left(w_{1} \widehat{\mathbf{w}_{1}}+w_{2} \widehat{\mathbf{w}_{2}}\right)=\left(1+w_{1} w_{2} \mathrm{e}^{-\widehat{\mathbf{w}_{3}} \phi}\right)^{-1}\left(w_{1}+w_{2} \mathrm{e}^{\widehat{\mathbf{w}_{3}} \phi}\right) \widehat{\mathbf{w}_{1}} .
$$

From this we can extract

$$
w_{1 \oplus 2} \mathrm{e}^{\widehat{\mathrm{w}_{3}} \phi_{1 \oplus 2}}=\left(1+w_{1} w_{2} \mathrm{e}^{-\widehat{\mathrm{w}_{3}} \phi}\right)^{-1}\left(w_{1}+w_{2} \mathrm{e}^{\widehat{\mathbf{w}_{3}} \phi}\right)=\frac{\left(w_{1}+w_{2} \mathrm{e}^{\widehat{\mathrm{w}_{3}} \phi}\right)}{\left(1+w_{1} w_{2} \mathrm{e}^{-\widehat{\mathbf{w}_{3}} \phi}\right)} .
$$

Thence

$$
w_{1 \oplus 2} \mathrm{e}^{\widehat{\mathrm{w}_{3}} \phi_{1 \oplus 2}}=\frac{\left(w_{1}+w_{2} \mathrm{e}^{\widehat{\mathrm{w}_{3}} \phi}\right)}{\left(1+w_{1} w_{2} \mathrm{e}^{-\widehat{\mathbf{w}_{3}} \phi}\right)} .
$$

This finally is a fully explicit result for general velocities $\vec{v}_{1}$ and $\vec{v}_{2}$, which is manifestly in agreement with the Giust-Vigoureux-Lages results [15].

\subsubsection{Uniqueness of the Composition Law}

Finally, we might note that the expression for the composition law (20) is not unique. For example, by considering the power-series of $\left(1-\mathbf{w}_{1} \mathbf{w}_{2}\right)^{-1}$, we can re-write Equation (20) as

$$
\mathbf{w}_{1 \oplus 2}=\left(1-\mathbf{w}_{1} \mathbf{w}_{2}\right)^{-1}\left(\mathbf{w}_{1}+\mathbf{w}_{2}\right)=\sum_{n=0}^{\infty}\left(\mathbf{w}_{1} \mathbf{w}_{2}\right)^{n}\left(\mathbf{w}_{1}+\mathbf{w}_{2}\right) .
$$

But, as $\mathbf{w}_{1}$ and $\mathbf{w}_{2}$ are pure quaternions, both $\mathbf{w}_{1}^{2}$ and $\mathbf{w}_{2}^{2}$ are real numbers, and so commute with $\mathbf{w}_{1}$ and $\mathbf{w}_{2}$. Thus,

$$
\mathbf{w}_{1 \oplus 2}=\sum_{n=0}^{\infty}\left(\mathbf{w}_{1} \mathbf{w}_{2}\right)^{n} \mathbf{w}_{1}+\sum_{n=0}^{\infty}\left(\mathbf{w}_{1} \mathbf{w}_{2}\right)^{n} \mathbf{w}_{2}=\mathbf{w}_{1} \sum_{n=0}^{\infty}\left(\mathbf{w}_{2} \mathbf{w}_{1}\right)^{n}+\mathbf{w}_{2} \sum_{n=0}^{\infty}\left(\mathbf{w}_{2} \mathbf{w}_{1}\right)^{n} .
$$

Consequently we find that our composition law can also be written as

$$
\mathbf{w}_{1 \oplus 2}=\left(\mathbf{w}_{1}+\mathbf{w}_{2}\right) \sum_{n=0}^{\infty}\left(\mathbf{w}_{2} \mathbf{w}_{1}\right)^{n}=\left(\mathbf{w}_{1}+\mathbf{w}_{2}\right)\left(1-\mathbf{w}_{2} \mathbf{w}_{1}\right)^{-1} .
$$

Indeed, one could use either Equation (20) or Equation (45) as the definition of the composition law $\oplus$. Nonetheless, we will stick with the convention given in (20). 


\subsection{Calculating the Wigner Angle}

In this section we obtain an expression for the Wigner angle for general 3-velocities using our composition law (20). Our calculations are obtained using the result that the Wigner angle is the angle between the velocities $\mathbf{w}_{1 \oplus 2}$ and $\mathbf{w}_{2 \oplus 1}$. We first note

$$
\left|\mathbf{w}_{1 \oplus 2}\right|=\left|\mathbf{w}_{2 \oplus 1}\right|=\left|1-\mathbf{w}_{1} \mathbf{w}_{2}\right|^{-1}\left|\mathbf{w}_{1}+\mathbf{w}_{2}\right|=\frac{\left|w_{1} \hat{\mathbf{n}}_{1}+w_{2} \hat{\mathbf{n}}_{2}\right|}{\left|1-w_{1} w_{2} \hat{\mathbf{n}}_{1} \hat{\mathbf{n}}_{2}\right|} .
$$

Thence, setting $\cos \theta=\vec{n}_{1} \cdot \vec{n}_{2}$ we explicitly verify

$$
\left|\mathbf{w}_{1 \oplus 2}\right|=\left|\mathbf{w}_{2 \oplus 1}\right|=\sqrt{\frac{w_{1}^{2}+w_{2}^{2}+2 w_{1} w_{2} \cos \theta}{1+w_{1}^{2} w_{2}^{2}+2 w_{1} w_{2} \cos \theta}} .
$$

Now note that because $\left|\mathbf{w}_{1 \oplus 2}\right|=\left|\mathbf{w}_{2 \oplus 1}\right|$ it follows that $\left(\mathbf{w}_{1 \oplus 2}\right)\left(\mathbf{w}_{2 \oplus 1}\right)^{-1}$ is a unit norm quaternion. In fact it is related to the Wigner angle by

$$
\mathrm{e}^{\Omega}=\left(\mathbf{w}_{1 \oplus 2}\right)\left(\mathbf{w}_{2 \oplus 1}\right)^{-1} .
$$

Then

$$
\mathrm{e}^{\Omega}=\left(\left(1-\mathbf{w}_{1} \mathbf{w}_{2}\right)^{-1}\left(\mathbf{w}_{1}+\mathbf{w}_{2}\right)\right)\left(\left(1-\mathbf{w}_{2} \mathbf{w}_{1}\right)^{-1}\left(\mathbf{w}_{2}+\mathbf{w}_{1}\right)\right)^{-1}
$$

But since for a product of quaternions $\left(\mathbf{q}_{1} \mathbf{q}_{2}\right)^{-1}=\mathbf{q}_{2}^{-1} \mathbf{q}_{1}^{-1}$ this reduces to

$$
\mathrm{e}^{\Omega}=\left(1-\mathbf{w}_{1} \mathbf{w}_{2}\right)^{-1}\left(1-\mathbf{w}_{2} \mathbf{w}_{1}\right) .
$$

Now

$$
\mathbf{w}_{1} \mathbf{w}_{2}=-w_{1} w_{2} \cos \theta+\left(\vec{w}_{1} \times \vec{w}_{2}\right) \cdot(\mathbf{i}, \mathbf{j}, \mathbf{k})
$$

Let us define

$$
\hat{\Omega}=\frac{\left(\vec{w}_{1} \times \vec{w}_{2}\right)}{\left|\vec{w}_{1} \times \vec{w}_{2}\right|} ; \quad \text { so } \quad \hat{w}_{1} \times \hat{w}_{2}=\sin \theta \hat{\Omega} .
$$

Then setting $\hat{\mathbf{\Omega}}=\hat{\Omega} \cdot(\mathbf{i}, \mathbf{j}, \mathbf{k})$ so that $\mathbf{\Omega}=\Omega \hat{\mathbf{\Omega}}$ we have:

$$
\mathbf{w}_{1} \mathbf{w}_{2}=-w_{1} w_{2}(\cos \theta-\sin \theta \hat{\mathbf{\Omega}})=-w_{1} w_{2} \mathrm{e}^{-\theta \hat{\mathbf{\Omega}}} .
$$

Consequently the Wigner angle satisfies

$$
\mathrm{e}^{\Omega}=\mathrm{e}^{\Omega \hat{\Omega}}=\left(1+w_{1} w_{2} \mathrm{e}^{-\theta \hat{\mathbf{\Omega}}}\right)^{-1}\left(1+w_{1} w_{2} \mathrm{e}^{\theta \hat{\mathbf{\Omega}}}\right)=\frac{1+w_{1} w_{2} \mathrm{e}^{\theta \hat{\mathbf{\Omega}}}}{1+w_{1} w_{2} \mathrm{e}^{-\theta \hat{\mathbf{\Omega}}}} .
$$

Equivalently,

$$
\mathrm{e}^{\Omega \hat{\Omega} / 2}=\frac{1+w_{1} w_{2} \mathrm{e}^{\theta \hat{\mathbf{\Omega}}}}{\mid 1+w_{1} w_{2} \mathrm{e}^{\theta \hat{\mathbf{\Omega}} \mid}} .
$$

Taking the scalar and vectorial parts of Equation (55), we finally obtain

$$
\tan \frac{\Omega}{2}=\frac{w_{1} w_{2} \sin \theta}{1+w_{1} w_{2} \cos \theta}=\frac{\left|\vec{w}_{1} \times \vec{w}_{2}\right|}{1+\vec{w}_{1} \cdot \vec{w}_{2}}
$$

as an explicit expression for the Wigner angle $\Omega$.

The simplicity of Equation (56) compared to existing formulae for $\Omega$ in the literature, shows how the composition law (20) can lead to much tidier and simpler formulae than other methods allowed for. This can be seen as the extension of the result (12) to more general velocities. 
We can write Equation (56) in a perhaps more familiar (though possibly more tedious) form by first noting that from Equation (27) we have

$$
w_{i}=\frac{1-\sqrt{1-v_{i}^{2}}}{v_{i}}=\frac{\gamma_{i}-1}{\sqrt{\gamma_{i}^{2}-1}}=\sqrt{\frac{\gamma_{i}-1}{\gamma_{i}+1}}=\frac{\sqrt{\gamma_{i}^{2}-1}}{\gamma_{i}+1}=\frac{v_{i} \gamma_{i}}{\gamma_{i}+1}
$$

and so

$$
\tan \frac{\Omega}{2}=\frac{v_{1} v_{2} \gamma_{1} \gamma_{2} \sin \theta}{\left(1+\gamma_{1}\right)\left(1+\gamma_{2}\right)+v_{1} v_{2} \gamma_{1} \gamma_{2} \cos \theta}
$$

We can check two interesting cases of Equation (56) for when $\theta=0$ (parallel velocities) and when $\theta=\pi / 2$ (perpendicular velocities). We can see directly that, for parallel velocities, the associated Wigner angle is given by $\tan (\Omega / 2)=0$, so that $\Omega=n \pi$ for $n \in \mathbb{Z}$; whilst for perpendicular velocities, the associated Wigner angle is simply given by $\tan (\Omega / 2)=w_{1} w_{2}$.

It is easiest to check our results against the literature using the somewhat messier Equation (58), in which case parallel velocities again give $\tan (\Omega / 2)=0$, whilst perpendicular velocities give

$$
\tan (\Omega / 2)=\frac{v_{1} v_{2} \gamma_{1} \gamma_{2}}{\left(1+\gamma_{1}\right)\left(1+\gamma_{2}\right)}
$$

which agrees with the results given in Reference [22].

\section{Combining Three 3-Velocities}

Let us now see what happens when we relativistically combine 3 half-velocities. We shall calculate, compare, and contrast $\mathbf{w}_{(1 \oplus 2) \oplus 3}$ with $\mathbf{w}_{1 \oplus(2 \oplus 3)}$.

\subsection{Combining 3 Half-Velocities: $\mathbf{w}_{(1 \oplus 2) \oplus 3}$}

Start from our key result

$$
\mathbf{w}_{1 \oplus 2}=\mathbf{w}_{1} \oplus \mathbf{w}_{2}=\left(1-\mathbf{w}_{1} \mathbf{w}_{2}\right)^{-1}\left(\mathbf{w}_{1}+\mathbf{w}_{2}\right)
$$

and iterate it to yield

$$
\mathbf{w}_{(1 \oplus 2) \oplus 3}=\left\{1-\left(1-\mathbf{w}_{1} \mathbf{w}_{2}\right)^{-1}\left(\mathbf{w}_{1}+\mathbf{w}_{2}\right) \mathbf{w}_{3}\right\}^{-1}\left\{\left(1-\mathbf{w}_{1} \mathbf{w}_{2}\right)^{-1}\left(\mathbf{w}_{1}+\mathbf{w}_{2}\right)+\mathbf{w}_{3}\right\}
$$

It is now a matter of straightforward quaternionic algebra to check that

$$
\begin{aligned}
\mathbf{w}_{(1 \oplus 2) \oplus 3}= & \left\{\left(1-\mathbf{w}_{1} \mathbf{w}_{2}\right)^{-1}\left(1-\mathbf{w}_{1} \mathbf{w}_{2}-\left(\mathbf{w}_{1}+\mathbf{w}_{2}\right) \mathbf{w}_{3}\right)\right\}^{-1} \\
\quad \times\left\{\left(1-\mathbf{w}_{1} \mathbf{w}_{2}\right)^{-1}\left(\mathbf{w}_{1}+\mathbf{w}_{2}\right)+\mathbf{w}_{3}\right\} & \\
= & \left(1-\mathbf{w}_{1} \mathbf{w}_{2}-\left(\mathbf{w}_{1}+\mathbf{w}_{2}\right) \mathbf{w}_{3}\right)^{-1}\left(1-\mathbf{w}_{1} \mathbf{w}_{2}\right)\left\{\left(1-\mathbf{w}_{1} \mathbf{w}_{2}\right)^{-1}\left(\mathbf{w}_{1}+\mathbf{w}_{2}\right)+\mathbf{w}_{3}\right\} \\
= & \left(1-\mathbf{w}_{1} \mathbf{w}_{2}-\left(\mathbf{w}_{1}+\mathbf{w}_{2}\right) \mathbf{w}_{3}\right)^{-1}\left\{\left(\mathbf{w}_{1}+\mathbf{w}_{2}\right)+\left(1-\mathbf{w}_{1} \mathbf{w}_{2}\right) \mathbf{w}_{3}\right\} .
\end{aligned}
$$

Ultimately we have the novel result

$$
\mathbf{w}_{(1 \oplus 2) \oplus 3}=\left\{1-\mathbf{w}_{1} \mathbf{w}_{2}-\mathbf{w}_{1} \mathbf{w}_{3}-\mathbf{w}_{2} \mathbf{w}_{3}\right\}^{-1}\left\{\mathbf{w}_{1}+\mathbf{w}_{2}+\mathbf{w}_{3}-\mathbf{w}_{1} \mathbf{w}_{2} \mathbf{w}_{3}\right\} .
$$

An alternative formulation starts from

$$
\mathbf{w}_{1 \oplus 2}=\mathbf{w}_{1} \oplus \mathbf{w}_{2}=\left(\mathbf{w}_{1}+\mathbf{w}_{2}\right)\left(1-\mathbf{w}_{2} \mathbf{w}_{1}\right)^{-1}
$$

which when iterated yields

$$
\mathbf{w}_{(1 \oplus 2) \oplus 3}=\left\{\left(\mathbf{w}_{1}+\mathbf{w}_{2}\right)\left(1-\mathbf{w}_{2} \mathbf{w}_{1}\right)^{-1}+\mathbf{w}_{3}\right\}\left\{1-\mathbf{w}_{3}\left(\mathbf{w}_{1}+\mathbf{w}_{2}\right)\left(1-\mathbf{w}_{2} \mathbf{w}_{1}\right)^{-1}\right\}^{-1} .
$$


Thence a little straightforward quaternionic algebra verifies that

$$
\begin{aligned}
& \mathbf{w}_{(1 \oplus 2) \oplus 3}=\left\{\left(\mathbf{w}_{1}+\mathbf{w}_{2}\right)+\mathbf{w}_{3}\left(1-\mathbf{w}_{2} \mathbf{w}_{1}\right)\right\}\left(1-\mathbf{w}_{2} \mathbf{w}_{1}\right)^{-1} \\
& \quad \times\left\{1-\mathbf{w}_{3}\left(\mathbf{w}_{1}+\mathbf{w}_{2}\right)\left(1-\mathbf{w}_{2} \mathbf{w}_{1}\right)^{-1}\right\}^{-1} \\
&=\left\{\left(\mathbf{w}_{1}+\mathbf{w}_{2}\right)+\mathbf{w}_{3}\left(1-\mathbf{w}_{2} \mathbf{w}_{1}\right)\right\}\left\{\left(1-\mathbf{w}_{2} \mathbf{w}_{1}\right)-\mathbf{w}_{3}\left(\mathbf{w}_{1}+\mathbf{w}_{2}\right)\right\}^{-1}
\end{aligned}
$$

Ultimately we have the novel result

$$
\mathbf{w}_{(1 \oplus 2) \oplus 3}=\left\{\mathbf{w}_{1}+\mathbf{w}_{2}+\mathbf{w}_{3}-\mathbf{w}_{3} \mathbf{w}_{2} \mathbf{w}_{1}\right\}\left\{1-\mathbf{w}_{2} \mathbf{w}_{1}-\mathbf{w}_{3} \mathbf{w}_{1}-\mathbf{w}_{3} \mathbf{w}_{2}\right\}^{-1} .
$$

So we have found two equivalent and novel formulae for $\mathbf{w}_{(1 \oplus 2) \oplus 3}$ Equations (63) and (67).

\subsection{Combining 3 Half-Velocities: $\mathbf{w}_{1 \oplus(2 \oplus 3)}$}

In contrast, the situation for $\mathbf{w}_{1 \oplus(2 \oplus 3)}$ is considerably more subtle. Start from the key result that

$$
\mathbf{w}_{2 \oplus 3}=\mathbf{w}_{2} \oplus \mathbf{w}_{3}=\left(1-\mathbf{w}_{2} \mathbf{w}_{3}\right)^{-1}\left(\mathbf{w}_{2}+\mathbf{w}_{3}\right),
$$

and iterate it to yield

$$
\mathbf{w}_{1 \oplus(2 \oplus 3)}=\left\{1-\mathbf{w}_{1}\left(1-\mathbf{w}_{2} \mathbf{w}_{3}\right)^{-1}\left(\mathbf{w}_{2}+\mathbf{w}_{3}\right)\right\}^{-1}\left\{\mathbf{w}_{1}+\left(1-\mathbf{w}_{2} \mathbf{w}_{3}\right)^{-1}\left(\mathbf{w}_{2}+\mathbf{w}_{3}\right)\right\} .
$$

The relevant quaternionic algebra is now a little trickier

$$
\begin{aligned}
\mathbf{w}_{1 \oplus(2 \oplus 3)}= & \left\{1-\mathbf{w}_{1}\left(1-\mathbf{w}_{2} \mathbf{w}_{3}\right)^{-1}\left(\mathbf{w}_{2}+\mathbf{w}_{3}\right)\right\}^{-1}\left(1-\mathbf{w}_{2} \mathbf{w}_{3}\right)^{-1} \\
= & \times\left\{\left(1-\mathbf{w}_{2} \mathbf{w}_{3}\right) \mathbf{w}_{1}+\left(\mathbf{w}_{2}+\mathbf{w}_{3}\right)\right\} \\
= & \left\{\left(1-\mathbf{w}_{2} \mathbf{w}_{3}\right)\left(1-\mathbf{w}_{1}\left(1-\mathbf{w}_{2} \mathbf{w}_{3}\right)^{-1}\left(\mathbf{w}_{2}+\mathbf{w}_{3}\right)\right\}^{-1}\right. \\
& \times\left\{\left(1-\mathbf{w}_{2} \mathbf{w}_{3}\right) \mathbf{w}_{1}+\left(\mathbf{w}_{2}+\mathbf{w}_{3}\right)\right\} \\
& \left.\times \mathbf{w}_{2} \mathbf{w}_{3}-\left(1-\mathbf{w}_{2} \mathbf{w}_{3}\right) \mathbf{w}_{1}\left(1-\mathbf{w}_{2} \mathbf{w}_{3}\right)^{-1}\left(\mathbf{w}_{2}+\mathbf{w}_{3}\right)\right\}^{-1} \\
& \times\left\{\mathbf{w}_{1}+\mathbf{w}_{2}+\mathbf{w}_{3}-\mathbf{w}_{2} \mathbf{w}_{3} \mathbf{w}_{1}\right\} .
\end{aligned}
$$

To proceed we note that

$$
\begin{aligned}
\left(1-\mathbf{w}_{2} \mathbf{w}_{3}\right) \mathbf{w}_{1}\left(1-\mathbf{w}_{2} \mathbf{w}_{3}\right)^{-1}= & \left(\frac{1-\mathbf{w}_{2} \mathbf{w}_{3}}{\left|1-\mathbf{w}_{2} \mathbf{w}_{3}\right|}\right) \mathbf{w}_{1}\left(\frac{1-\mathbf{w}_{2} \mathbf{w}_{3}}{\left|1-\mathbf{w}_{2} \mathbf{w}_{3}\right|}\right)^{-1} \\
& =e^{-\mathbf{\Omega}_{2 \oplus 3} / 2} \mathbf{w}_{1} e^{+\boldsymbol{\Omega}_{2 \oplus 3} / 2} .
\end{aligned}
$$

Thence we have the novel result

$$
\mathbf{w}_{1 \oplus(2 \oplus 3)}=\left\{1-\mathbf{w}_{2} \mathbf{w}_{3}-\left(e^{-\Omega_{2 \oplus 3} / 2} \mathbf{w}_{1} e^{+\Omega_{2 \oplus 3} / 2}\right)\left(\mathbf{w}_{2}+\mathbf{w}_{3}\right)\right\}^{-1}\left\{\mathbf{w}_{1}+\mathbf{w}_{2}+\mathbf{w}_{3}-\mathbf{w}_{2} \mathbf{w}_{3} \mathbf{w}_{1}\right\} .
$$

While structurally similar to the formulae (63) and (67) for $\mathbf{w}_{(1 \oplus 2) \oplus 3}$ the present result (72) for $\mathbf{w}_{1 \oplus(2 \oplus 3)}$ is certainly different-the Wigner angle $\boldsymbol{\Omega}_{2 \oplus 3}$ now makes an explicit appearance, also the form of the triple-product $\mathbf{w}_{2} \mathbf{w}_{3} \mathbf{w}_{1}$ is different.

\subsection{Combining 3 Half-Velocities: (Non)-Associativity}

From (63) and (67) for $\mathbf{w}_{(1 \oplus 2) \oplus 3}$, and (72) for $\mathbf{w}_{1 \oplus(2 \oplus 3)}$, it is clear that relativistic composition of velocities is in general not associative. (See for instance the discussion in References [28,29], commenting on Reference [29].) A sufficient condition for associativity, $\mathbf{w}_{(1 \oplus 2) \oplus 3}=\mathbf{w}_{1 \oplus(2 \oplus 3)}$, is to enforce

$$
e^{-\Omega_{2 \oplus 3} / 2} \mathbf{w}_{1} e^{+\Omega_{2 \oplus 3} / 2}=\mathbf{w}_{1} ; \quad \text { and } \quad \mathbf{w}_{1} \mathbf{w}_{2} \mathbf{w}_{3}=\mathbf{w}_{2} \mathbf{w}_{3} \mathbf{w}_{1} .
$$


That is, a sufficient condition for associativity is

$$
\left[\mathbf{\Omega}_{2 \oplus 3}, \mathbf{w}_{1}\right]=0 ; \quad \text { and } \quad\left[\mathbf{w}_{1}, \mathbf{w}_{2} \mathbf{w}_{3}\right]=0 .
$$

But note $\boldsymbol{\Omega}_{2 \oplus 3} \propto\left[\mathbf{w}_{2}, \mathbf{w}_{3}\right]$ and $\mathbf{w}_{2} \mathbf{w}_{3}=\frac{1}{2}\left\{\mathbf{w}_{2}, \mathbf{w}_{3}\right\}+\frac{1}{2}\left[\mathbf{w}_{2}, \mathbf{w}_{3}\right]$. Since $\left\{\mathbf{w}_{2}, \mathbf{w}_{3}\right\} \in \mathbb{R}$, we then have $\left[\mathbf{w}_{1}, \mathbf{w}_{2} \mathbf{w}_{3}\right]=\frac{1}{2}\left[\mathbf{w}_{1},\left[\mathbf{w}_{2}, \mathbf{w}_{3}\right]\right]$. This now implies that these two sufficiency conditions are in fact identical; so a sufficient condition for associativity is

$$
\left[\mathbf{w}_{1},\left[\mathbf{w}_{2}, \mathbf{w}_{3}\right]\right]=0 .
$$

This sufficient condition for associativity can also be written as the vanishing of the vector triple product

$$
\vec{w}_{1} \times\left(\vec{w}_{2} \times \vec{w}_{3}\right)=0 .
$$

Equivalently

$$
\vec{v}_{1} \times\left(\vec{v}_{2} \times \vec{v}_{3}\right)=0
$$

\subsection{Specific Non-Coplanar Example}

As a final example of the power of the quaternion formalism, let us consider a specific intrinsically non-coplanar example. Let $\mathbf{w}_{1}=w_{1} \mathbf{i}, \mathbf{w}_{2}=w_{2} \mathbf{j}$, and $\mathbf{w}_{3}=w_{3} \mathbf{k}$ be three mutually perpendicular half-velocities. (So this configuration does automatically satisfy the associativity condition discussed above.) Then we have already seen that:

$$
\mathbf{w}_{1} \oplus \mathbf{w}_{2}=\frac{w_{1}\left(1-w_{2}^{2}\right) \mathbf{i}+w_{2}\left(1+w_{1}^{2}\right) \mathbf{j}}{1+w_{1}^{2} w_{2}^{2}} ; \quad w_{1 \oplus 2}^{2}=\frac{w_{1}^{2}+w_{2}^{2}}{1+w_{1}^{2} w_{2}^{2}} .
$$

Furthermore, since $\mathbf{w}_{1} \oplus \mathbf{w}_{2}$ is perpendicular to $\mathbf{w}_{3}$, we have

$$
\left(\mathbf{w}_{1} \oplus \mathbf{w}_{2}\right) \oplus \mathbf{w}_{3}=\frac{w_{1 \oplus 2}\left(1-w_{3}^{2}\right) \hat{\mathbf{n}}_{1 \oplus 2}+w_{3}\left(1+w_{1 \oplus 2}^{2}\right) \mathbf{k}}{1+w_{1 \oplus 2}^{2} w_{3}^{2}},
$$

and

$$
w_{(1 \oplus 2) \oplus 3}^{2}=\frac{w_{(1 \oplus 2)}^{2}+w_{3}^{2}}{1+w_{(1 \oplus 2)}^{2} w_{3}^{2}}=\frac{w_{1}^{2}+w_{2}^{2}+w_{3}^{2}+w_{1}^{2} w_{2}^{2} w_{3}^{2}}{1+w_{1}^{2} w_{2}^{2}+w_{2}^{2} w_{3}^{2}+w_{3}^{2} w_{1}^{2}} .
$$

A little algebra now yields the manifestly non-commutative result

$$
\left(\mathbf{w}_{1} \oplus \mathbf{w}_{2}\right) \oplus \mathbf{w}_{3}=\frac{\left(1-w_{2}^{2}\right)\left(1-w_{3}^{2}\right) \mathbf{w}_{1}+\left(1+w_{1}^{2}\right)\left(1-w_{3}^{2}\right) \mathbf{w}_{2}+\left(1+w_{1}^{2}\right)\left(1+w_{2}^{2}\right) \mathbf{w}_{3}}{1+w_{1}^{2} w_{2}^{2}+w_{2}^{2} w_{3}^{2}+w_{3}^{2} w_{1}^{2}} .
$$

In this particular case we can also explicitly show that

$$
\left(\mathbf{w}_{1} \oplus \mathbf{w}_{2}\right) \oplus \mathbf{w}_{3}=\mathbf{w}_{1} \oplus\left(\mathbf{w}_{2} \oplus \mathbf{w}_{3}\right),
$$

though (as discussed above) associativity fails in general.

\section{Conclusions}

Herein we have provided a simple, elegant, and novel algebraic method for combining special relativistic 3-velocities using quaternions:

$$
\mathbf{w}_{1 \oplus 2}=\mathbf{w}_{1} \oplus \mathbf{w}_{2}=\left(1-\mathbf{w}_{1} \mathbf{w}_{2}\right)^{-1}\left(\mathbf{w}_{1}+\mathbf{w}_{2}\right)=\left(\mathbf{w}_{1}+\mathbf{w}_{2}\right)\left(1-\mathbf{w}_{2} \mathbf{w}_{1}\right)^{-1} .
$$


The construction also leads to a simple, elegant, and novel formula for the Wigner angle:

$$
e^{\Omega}=e^{\Omega \hat{\Omega}}=\left(1-\mathbf{w}_{1} \mathbf{w}_{2}\right)^{-1}\left(1-\mathbf{w}_{2} \mathbf{w}_{1}\right),
$$

in terms of which

$$
\hat{\mathbf{w}}_{1 \oplus 2}=\mathrm{e}^{\Omega / 2} \frac{\mathbf{w}_{1}+\mathbf{w}_{2}}{\left|\mathbf{w}_{1}+\mathbf{w}_{2}\right|} ; \quad \hat{\mathbf{w}}_{2 \oplus 1}=\mathrm{e}^{-\Omega / 2} \frac{\mathbf{w}_{1}+\mathbf{w}_{2}}{\left|\mathbf{w}_{1}+\mathbf{w}_{2}\right|} .
$$

All of the non-commutativity associated with non-collinearity of 3-velocities is automatically and rather efficiently dealt with by the quaternion algebra.

Author Contributions: Conceptualization, T.B. and M.V.; methodology, T.B. and M.V.; software, T.B. and M.V.; validation, T.B. and M.V.; formal analysis, T.B. and M.V.; resources, M.V.; writing-original draft preparation, T.B. and M.V.; writing-review and editing, T.B. and M.V.; supervision, M.V.; project administration, M.V.; funding acquisition, M.V. All authors have read and agreed to the published version of the manuscript.

Funding: T.B. was supported by a Victoria University of Wellington MSc scholarship, and was also indirectly supported by the Marsden Fund, via a grant administered by the Royal Society of New Zealand. M.V. was directly supported by the Marsden Fund, via a grant administered by the Royal Society of New Zealand.

Conflicts of Interest: The authors declare no conflict of interest.

\section{References}

1. Silberstein, L. Quaternionic form of relativity. Philisophical Mag. 1912, 23, 790-809. [CrossRef]

2. Silberstein, L. The Theory of Relativity; Macmillan and Co: London, UK, 1914

3. Silberstein, L. Available online: https://en.wikipedia.org/wiki/Ludwik_Silberstein (accessed on 8 December 2020).

4. Dirac, P.A.M. Application of quaternions to Lorentz transformations. Proc. R. Ir. Acad. 1944, 50, $261-270$.

5. Rastall, P. Quaternions in Relativity. Rev. Mod. Phys. 1964, 36, 820. [CrossRef]

6. Girard, P.R. The quaternion group and modern physics. Eur. J. Phys. 1984, 5, 25-32. [CrossRef]

7. Ungar, A.A. The relativistic velocity composition paradox and the Thomas rotation. Found. Phys. 1989, 19, 1385-1396. [CrossRef]

8. Mocanu, C.I. On the relativistic velocity composition paradox and the Thomas rotation. Found. Phys. Lett. 1992, 5, 443-456. [CrossRef]

9. De Leo, S. Quaternions and Special Relativity. J. Math. Phys. 1996, 37, 2955-2968. [CrossRef]

10. Friedman, Y. Physical Applications of Homogeneous Balls; Progress in Mathematical Physics; Springer: Birkhäuser, Basel, 2005; Volume 40, doi:10.1007/978-0-8176-8208-8. [CrossRef]

11. Friedman, Y.; Semon, M.D. Relativistic acceleration of charged particles in uniform and mutually perpendicular electric and magnetic fields as viewed in the laboratory frame. Phys. Rev. E 2005, 72, 026603. [CrossRef]

12. Greiter, M.; Schuricht, D. Imaginary in all directions: An Elegant formulation of special relativity and classical electrodynamics. Eur. J. Phys. 2003, 24, 397-401. [CrossRef]

13. Yefremov, A.P. Theory of relativity in quaternion spinors. Gravit. Cosmol. 2016, 22, 97-106. [CrossRef]

14. Wigner, E. On unitary representations of the inhomogeneous Lorentz group. Ann. Math. 1939, 40, $149-204$. [CrossRef]

15. Giust, R.; Vigoureux, J.-M.; Lages, J. Generalized composition law from $2 \times 2$ matrices. Am. J. Phys. 2009, 77, 1068-1073. [CrossRef]

16. Lages, J.; Giust, R.; Vigoureux, J.-M. Composition law for polarizers. Phys. Rev. 2008, 78, 033810. [CrossRef]

17. Thomas, L.H. The motion of the spinning electron. Nature 1926, 117, 514-514. [CrossRef]

18. Fisher, G.P. Thomas precession. Am. J. Phys. 1972, 40, 1772. [CrossRef]

19. Ferraro, M.; Thibeault, R. Generic composition of boosts: An elementary derivation of the Wigner rotation. Eur. J. Phys. 1999, 20, 143. [CrossRef]

20. Malykin, G.B. Thomas precession: Correct and incorrect solutions. Phys. Uspekhi 2006, 49, 837-853. [CrossRef]

21. Ritus, V.I. On the difference between Wigner's and Møller's approaches to the description of Thomas precession. Phys. Uspekhi 2007, 50, 95-101. [CrossRef] 
22. O'Donnell, K.; Visser, M. Elementary analysis of the special relativistic combination of velocities, Wigner rotation, and Thomas precession. Eur. J. Phys. 2011, 32, 1033-1047. [CrossRef]

23. Achilles, R.; Bonfiglioli, A. The early proofs of the theorem of Campbell, Baker, Hausdorff, and Dynkin. Arch. Hist. Exact Sci. 2012, 66, 295-358. [CrossRef]

24. Goldberg, K. The formal power series for $\log \left(e^{x} e^{y}\right)$. Duke Math. J. 1956, 23, 13-21. [CrossRef]

25. Van-Brunt, A.; Visser, M. Special-case closed form of the Baker-Campbell-Hausdorff formula. J. Phys. A 2015, 48, 225207. [CrossRef]

26. Van-Brunt, A.; Visser, M. Simplifying the Reinsch algorithm for the Baker-Campbell-Hausdorff series. J. Math. Phys. 2016, 57, 023507. [CrossRef]

27. Van-Brunt, A.; Visser, M. Explicit Baker-Campbell-Hausdorff Expansions. Mathematics 2018, 6, 135. [CrossRef]

28. Ungar, A.A. Thomas precession: A kinematic effect of the algebra of Einstein's velocity addition law. Comments on 'Deriving relativistic momentum and energy: II. Three-dimensional case'. Eur. J. Phys. 2006, 27, L17. [CrossRef]

29. Sonego, S.; Pin, M. Deriving relativistic momentum and energy: II. Three-dimensional case (CORRIGENDUM). Eur. J. Phys. 2005, 26, 851-856, Correction in 2006, 27, 685. [CrossRef]

Publisher's Note: MDPI stays neutral with regard to jurisdictional claims in published maps and institutional affiliations.

(C) 2020 by the authors. Licensee MDPI, Basel, Switzerland. This article is an open access article distributed under the terms and conditions of the Creative Commons Attribution (CC BY) license (http:/ / creativecommons.org/licenses/by/4.0/). 